\title{
Success probability of integer GPS ambiguity rounding and bootstrapping
}

\author{
P. J. G. Teunissen \\ Delft Geodetic Computing Centre (LGR), Faculty of Geodesy, Delft University of Technology, Thijsseweg 11, 2629 JA, The Netherlands \\ E-mail: P.J.G. Teunissen@geo.tudelft.nl
}

Received: 24 March 1998 / Accepted: 8 June 1998

\begin{abstract}
Global Positioning System ambiguity resolution is usually based on the integer least-squares principle (Teunissen 1993). Solution of the integer least-squares problem requires both the execution of a search process and an ambiguity decorrelation step to enhance the efficiency of this search. Instead of opting for the integer least-squares principle, one might also want to consider less optimal integer solutions, such as those obtained through rounding or sequential rounding. Although these solutions are less optimal, they do have one advantage over the integer least-squares solution: they do not require a search and can therefore be computed directly. However, in order to be confident that these less optimal solutions are still good enough for the application at hand, one requires diagnostic measures to predict their rate of success. These measures of confidence are presented and it is shown how they can be computed and evaluated.
\end{abstract}

Key words. GPS ambiguity · Probability · Integer rounding $\cdot$ Integer bootstrapping

\section{Introduction}

Global Positioning System (GPS) ambiguity resolution, which is the process of resolving the unknown cycle ambiguities of the double-difference (DD) carrier phase data as integers, consists of an estimation part and a validation part. The purpose of the validation part is to infer, given the data set at hand, whether or not the actual integer solution is sufficiently consistent with the GPS model used. The purpose of the estimation part, on the other hand, is to come up with a procedure for obtaining an integer solution and to show which quality this solution is likely to have. In other words, for the estimation part one has to decide which integer estima- tor to use, as well as provide a description of its qualitative properties.

Although GPS research in the last decade or so has resulted in a variety of different methods and proposals for estimating integer ambiguities, there is a growing consensus that the use of the integer least-squares principle is the preferred approach for integer ambiguity estimation. There are two main reasons for this. First, the integer least-squares estimator is optimal in the sense that it maps the largest probability mass onto the integer vector of ambiguity means. Second, it has been demonstrated that by means of a least-squares ambiguity decorrelation adjustment, it is now possible to compute the integer least-squares solution rigorously and efficiently. The pitfalls associated with some of the more classical approaches can thus be avoided. For a discussion of these pitfalls, see Teunissen (1997a).

When the ambiguity variance-covariance matrix is diagonal, the integer least-squares estimator reduces to the estimator that corresponds to a simple componentwise rounding of the real-valued least-squares ambiguity vector. This is not the case, however, when the ambiguity variance-covariance matrix is non-diagonal. Nevertheless, in this case one could still decide to stick to the simple rounding mechanism in order to obtain an integer solution. Although the integer estimator so obtained will be less optimal, it might still be 'optimal enough' for the particular application at hand. This requires, however, that one is also able to predict the success rate of this simple integer estimator. Hence, in order to evaluate the quality of this estimator, we need to be able to evaluate its probability of correct integer estimation. It is the purpose of this contribution to present these measures of confidence for two simple integer estimators, the one that corresponds to a componentwise rounding and the one that corresponds to a sequential rounding of the individual ambiguities.

In Sect. 2 we first give a brief overview of the estimation steps involved in ambiguity resolution. In this section we also illustrate how to set the size of the ambiguity search space. The integer vector obtained by 
means of either simple rounding or a sequential rounding is particularly suited for this, provided the ambiguity decorrelation process precedes the rounding step. In Sect. 3, we present diagnostic measures to evaluate whether or not it makes sense to use the simple rounding mechanism in order to obtain the integer ambiguity vector. In Sect. 4 this is also done for the integer ambiguity vector obtained through a sequential rounding. We also prove that the probability of correct integer estimation based on a sequential rounding is larger than, or at least as large as, the probability of correct integer estimation based on a straightforward rounding. Finally, we give an upper bound for the probability of correct integer estimation which is invariant for the whole class of admissible ambiguity transformations. It is based on the determinant of the ambiguity variance-covariance matrix, for which closed-form expressions are available for a variety of different GPS models.

\section{The ambiguity search space}

\subsection{Integer least-squares estimation}

In principle, all the GPS models can be cast in the following conceptual frame of linear(ized) observation equations:

$y=A a+B b+e$

where $y$ is the given data vector, $a$ and $b$ are the unknown parameter vectors of order $n$ and $p$, respectively, and $e$ is the noise vector of order $m$. The matrices $A$ and $B$ are the corresponding design matrices of order $m \times n$ and $m \times p$ respectively. The data vector $y$ will usually consist of the 'observed minus computed' singleor dual-frequency DD phase and/or pseudo range (code) observations, accumulated over all observation epochs. The entries of vector $a$ are then the DD carrier phase ambiguities, expressed in units of cycles rather than range. They are known to be integers. The entries of vector $b$ will then consist of the remaining unknown parameters such as, for example, baseline components (coordinates) and possibly atmospheric delay parameters (troposphere, ionosphere). Note that we have followed the customary practice of using the DD version of the code and carrier phase observation equations. This is, however, not strictly needed. As an alternative one can also work with undifferenced or single-differenced observations (de Jonge 1998). In that case the non-integer ambiguities will have to be reparametrized so as to obtain integer ambiguities again.

When using the least-squares principle, the above system of observation equations can be solved by means of the minimization problem

$$
\begin{aligned}
& \min _{a, b}(y-A a-B b)^{T} Q_{y}^{-1}(y-A a-B b), \\
& a=\text { integer, } \quad b=\text { real }
\end{aligned}
$$

with $Q_{y}$ the variance-covariance matrix of the observables. This is a non-standard least-squares problem, due to the integer constraints on the ambiguities. This type of least-squares problem was first introduced by Teunissen (1993) and has been coined 'integer least-squares'. Conceptually, one can divide the computation of Eq. (2) into three different steps. In the first step one simply disregards the integer constraints on the ambiguities and performs a standard least-squares adjustment. As a result one obtains the (real-valued) least-squares estimates of $a$ and $b$, together with their variance-covariance matrix

$$
\left[\begin{array}{l}
\hat{a} \\
\hat{b}
\end{array}\right] ; \quad\left[\begin{array}{cc}
Q_{\hat{a}} & Q_{\hat{a} \hat{b}} \\
Q_{\hat{b} \hat{a}} & Q_{\hat{b}}
\end{array}\right]
$$

This solution is often referred to as the 'float' solution. If we assume that the noise vector $e$ of Eq. (1) is normally distributed with zero mean and variance-covariance matrix $Q_{y}$, then $\hat{a}$ is normally distributed with integer mean $a$ and variance-covariance matrix $Q_{\hat{a}}$. Thus

$\hat{a} \sim \mathrm{N}\left(a, Q_{\hat{a}}\right), \quad a=$ integer

In the second step the 'float' ambiguity estimate $\hat{a}$ and its variance-covariance matrix are used to compute the corresponding integer ambiguity estimate. This implies that one has to solve the minimization problem

$\min _{a}(\hat{a}-a)^{T} Q_{\hat{a}}^{-1}(\hat{a}-a), \quad a=$ integer

Its solution will be denoted as $\breve{a}$. Since the mapping from $\hat{a}$ to $\breve{a}$ is nonlinear, the distribution of the integer leastsquares estimator will no longer be normal (Gaussian). In fact, due to the mapping involved, it generally becomes very difficult to compute the exact distribution. It can be shown, however, that it is an admissible and min-max estimator. Approximations or bounds can be obtained for its probability of correct integer estimation. Examples can be found in works by Teunissen et al. (1996) and Teunissen $(1997 b, c)$ and also in Sect. 4 of the present paper.

Once the integer ambiguities are computed, they are finally used in the third step to correct the 'float' estimate of $b$. As a result one obtains the 'fixed' solution

$\breve{b}=\hat{b}-Q_{\hat{b} \hat{a}} Q_{\hat{a}}^{-1}(\hat{a}-\breve{a})$

The unbiasedness of the integer least-squares estimator was proven by Teunissen (1998). This implies that, since the 'float' solutions are also unbiased

$E\{\breve{a}\}=E\{\hat{a}\}=a \quad$ and $\quad E\{\breve{b}\}=E\{\hat{b}\}=b$

with $E\{\cdot\}$ the expectation operator. However, the 'fixed' solution will not be normally distributed, but it is approximately normal when the probability of correct integer estimation is sufficiently close to one. In that case, the 'fixed' solution also inherits the very high precision that corresponds with the case that the integer ambiguities are non-stochastic. In fact, this is the whole principle on which GPS ambiguity resolution is based.

From a computational point of view, the most difficult part in the above three steps is the solution of Eq. (5). It requires the minimization of a quadratic form 
over the whole $n$-dimensional space of integers. In order to tackle this problem, we first replace the whole space of integers by a smaller set of integers. This is the socalled ambiguity search space. It is in this local space that the search for the integer least-squares solution is performed. In the case of the GPS, however, this search is seriously hindered by the fact that the (real-valued) least-squares ambiguities are usually highly correlated. To remedy this situation, the least-squares ambiguity decorrelation adjustment (LAMBDA) was introduced (Teunissen 1993); for details of its performance see, e.g. de Jonge and Tiberius (1996) Boon and Ambrosius (1997), Jonkman (1998), Teunissen et al. (1997). By using this method, the original DD ambiguities are replaced by a set of transformed ambiguities which have the property of being very precise and largely decorrelated; see also the textbooks of Kleusberg and Teunissen (1996), Hofmann-Wellenhof et al. (1997) and Strang and Borre (1997). The ambiguity decorrelation process has the very beneficial effect of moulding the search space such that it transforms from a highly elongated ellipsoid to one that corresponds more closely to a sphere.

\subsection{Setting the size of the ambiguity search space}

The ambiguity search space is defined as the set of gridpoints $a$ that satisfy

$(\hat{a}-a)^{T} Q_{\hat{a}}^{-1}(\hat{a}-a) \leq \chi^{2}$

The positive constant $\chi^{2}$ is chosen such that it guarantees that the search space contains the solution sought. This can be achieved by computing the constant as

$\chi^{2}=\left(\hat{a}-a^{0}\right)^{T} Q_{\hat{a}}^{-1}\left(\hat{a}-a^{0}\right)$

in which $a^{0}$ is an arbitrary integer vector. However, in order to avoid the search space containing an abundance of unnecessary grid points, it helps if the constant can be chosen such that the size of the search space becomes small, while at the same time it remains guaranteed that it contains the integer least-squares solution sought. For that purpose $a^{0}$ should be a sufficiently good approximation to the integer least-squares solution. It may occasionally even happen that this approximation coincides with the integer least-squares solution. Of course, if this happens, a further search is no longer needed. A simple check as to whether the approximate solution coincides with the integer least-squares solution can be performed as follows. Using the cosine-rulebased decomposition

$$
\begin{aligned}
\left\|\hat{a}-a^{0}+\nabla\right\|^{2}= & \left\|\hat{a}-a^{0}\right\|^{2}+\|\nabla\|^{2} \\
& +2\left\|\hat{a}-a^{0}\right\|\|\nabla\| \cos \alpha
\end{aligned}
$$

with $\alpha$ the angle between $\hat{a}-a^{0}$ and the integer vector $\nabla$ and the norm taken with respect to the metric defined by the ambiguity variance-covariance matrix, it follows that $a^{0}$ will coincide with the integer least-squares solution when the sum of the last two terms is larger than or equal to zero for any non-zero integer vector $\nabla$.
This will certainly be the case when $\|\nabla\| \geq 2\left\|\hat{a}-a^{0}\right\|$ and thus also when $1 / \sqrt{ } \lambda_{\max } \geq 2\left\|\hat{a}-a^{0}\right\|$, where $\lambda_{\max }$ equals the largest eigenvalue of the ambiguity variancecovariance matrix. Hence, a sufficient check to see whether $a^{0}$ equals the integer least-squares solution is to check whether the reciprocal of the square root of the largest eigenvalue is larger than or equal to twice the distance between $\hat{a}$ and $a^{0}$. In order to obtain looser upper bounds, one may also replace the largest eigenvalue by the largest variance of $\hat{a}_{i}$ or by the largest sequential conditional variance.

There are two easy methods of computing an approximate integer solution. The first involves applying a componentwise rounding to the entries of $\hat{a}$ and the second a sequential rounding to the entries of $\hat{a}$. Results that show how well these approximate solutions perform in setting the size of the search space can be found in Teunissen et al. (1996) and de Jonge and Tiberius (1996). As is shown in these studies, the approximate solutions perform far better when they are preceded by the ambiguity decorrelation process. This also holds true for their respective probabilities of correct integer estimation. These probabilities will be developed in the following sections.

\section{Integer ambiguity rounding}

The simplest method of obtaining an approximation to the integer least-squares solution is to apply a componentwise rounding scheme to the entries of $\hat{a}$. This approximation may be used to set the size of the search space, but it can also be used as an integer estimator in its own right. Although it will be less optimal than the integer least-squares estimator, for particular applications at hand it may still perform sufficiently well. Diagnostics to evaluate whether or not this is the case are presented in this section.

\subsection{The scalar case}

Let us start with the simplest case that the 'float' solution $\hat{a}$ is a scalar. Thus we assume that there is only one single ambiguity. If we denote 'rounding to the nearest integer' by '[.]', the integer nearest to $\hat{a}$ reads

$\breve{a}_{R}=[\hat{a}]$

where the subscript ' $R$ ' refers to the fact that the integer is obtained through rounding. The probability mass function of this integer estimator is given as

$P\left(\breve{a}_{R}=i\right)=\int_{(i-a)-\frac{1}{2}}^{(i-a)+\frac{1}{2}} \frac{1}{\sqrt{2 \pi} \sigma_{\hat{a}}} \exp \left\{-\frac{1}{2} \hat{a}^{2} / \sigma_{\hat{a}}^{2}\right\} \mathrm{d} \hat{a}$

where $i$ ranges over the set of integers and $\sigma_{\hat{a}}$ denotes the standard deviation of $\hat{a}$. Hence, the probabilities are obtained through an integration of the normal distribution over intervals of length 1 which are all centred at an integer. Since the normal distribution is symmetric about its mean and since the mean of $\hat{a}$ is an integer, it 
immediately follows that the above integer estimator is unbiased, i.e. its expectation equals the integer $a$.

In order to infer the quality of the integer estimator for the purpose of ambiguity resolution, we require the probability of the event that Eq. (8) coincides with the true but unknown integer ambiguity $a$. This probability reads as

$P\left(\breve{a}_{R}=a\right)=P\left(|\hat{a}-a| \leq \frac{1}{2}\right)$

This probability can be evaluated by means of Eq. (9). The probability of rounding to the correct integer value then becomes

$P\left(\breve{a}_{R}=a\right)=2 \Phi\left(\frac{1}{2 \sigma_{\hat{a}}}\right)-1$

with

$\Phi(x)=\int_{-\infty}^{x} \frac{1}{\sqrt{2 \pi}} \exp \left(-\frac{1}{2} z^{2}\right) \mathrm{d} z$

Note that the probability of rounding to the correct integer value increases as the standard deviation of $\hat{a}$ becomes smaller. This is also what one would expect. By evaluating Eq. (11) and checking whether the probability is sufficiently close to 1 , one can now make the decision as to whether or not to treat the rounded ambiguity as being deterministic.

Although the computation of Eq. (11) by means of the function $\Phi(x)$ is straightforward, it is also helpful if we can approximate the probability by means of simple analytical expressions. There are several such approximations, one of which is

$\sqrt{1-\exp \left\{-\frac{1}{2} x^{2}\right\}} \leq P\left(\breve{a}_{R}=a\right) \leq \sqrt{1-\exp \left\{-x^{2}\right\}}$

with $x=1 / 2 \sigma_{\hat{a}}$; see e.g. Johnson et al. (1994).

\subsection{The vectorial case}

We now assume that $\hat{a}$ is a vector of order $n$, normally distributed with integer mean $a$ and variance-covariance matrix $Q_{\hat{a}}$. Componentwise rounding to the nearest integer gives the integer ambiguity vector

$\breve{a}_{R}=\left(\left[\hat{a}_{1}\right], \ldots,\left[\hat{a}_{n}\right]\right)^{T}$

The probability of rounding to the correct integer ambiguity vector $a$ now reads

$P\left(\breve{a}_{R}=a\right)=P\left(\cap_{i=1}^{n}\left\{\left|\hat{a}_{i}-a_{i}\right| \leq \frac{1}{2}\right\}\right)$

It is the probability that $\hat{a}$ lies in the $n$-dimensional cube, centred at $a$ and having sides of length 1. This probability is easy to evaluate when the ambiguities are fully decorrelated, i.e. when the ambiguity variance matrix is diagonal. In that case the problem decouples into $n$ independent scalar problems of the type of Eq. (10). The probability Eq. (13) then equals the product of $n$ probabilities of the type of Eq. (11). The exact evaluation of the probability Eq. (13) becomes very difficult when the ambiguity variance-covariance matrix is non-diagonal. Unfortunately, this is the case in actual practice. However, although it is difficult to evaluate Eq. (13) exactly in the correlated case, it is possible to formulate a lower bound for it. This lower bound is given by the probability corresponding to the decorrelated case. Thus we have

$\prod_{i=1}^{n}\left(2 \Phi\left(\frac{1}{2 \sigma_{\hat{a}_{i}}}\right)-1\right) \leq P\left(\breve{a}_{R}=a\right)$

This lower bound can now be used to check whether the simple estimator 'round to the nearest integer' guarantees sufficient success of obtaining the correct integer ambiguity vector. One simply has to evaluate the lower bound and check whether it is sufficiently close to 1 .

Note that the lower bound is only dependent on the diagonal entries of the ambiguity variance-covariance matrix. Hence, this lower bound is not invariant for the class of admissible ambiguity transformations (Teunissen 1995). Since the precision of the individual DD ambiguities is usually rather poor, the lower bound of Eq. (14) will usually be rather loose when applied to the DD ambiguities. However, the lower bound becomes much sharper when it is applied to ambiguities which are almost decorrelated. This shows that, for an actual application, the above lower bound should be evaluated for the decorrelated ambiguities obtained through the LAMBDA method. Since the transformed ambiguities obtained by this method are far more precise than the original DD ambiguities, the lower bound becomes sharper due to its increase in value.

\section{Integer ambiguity bootstrapping}

Another easy method of obtaining an approximation to the integer least-squares solution is to apply a sequential rounding scheme to the entries of $\hat{a}$. This approximation may also be used as an integer estimator in its own right. In this section we will present its probability of correct integer estimation and show how it is related to the results of the previous section.

\subsection{Probability of correct integer estimation}

The integer estimation scheme of componentwise rounding does not take the ambiguity correlations into account. It simply treats the multivariate integer estimation problem as if it were a problem consisting of $n$ independent scalar estimation problems. A method which does take some of the correlation into account is the 'sequential integer rounding' method, which also is referred to as the 'integer bootstrapping' method, see e.g. Blewitt (1989) and Dong and Bock (1989). This method is a generalization of the 'integer rounding' method, and it goes as follows. If $n$ ambiguities are available, one starts with the first ambiguity $\hat{a}_{1}$ and, as before, rounds its value to the nearest integer. Having obtained the integer value of this first ambiguity, the real-valued estimates of all remaining ambiguities are 
then corrected by virtue of their correlation with the first ambiguity. Then the second, but now corrected, realvalued ambiguity estimate is rounded to its nearest integer. Having obtained the integer value of the second ambiguity, the real-valued estimates of all remaining $n-2$ ambiguities are then again corrected, but now by virtue of their correlation with the second ambiguity. This process is continued until all ambiguities are taken care of. In essence this 'bootstrapping' technique boils down to the use of a sequential conditional least-squares adjustment (Teunissen 1993, 1996), with a conditioning on the integer ambiguity values obtained in the previous steps. The components of the integer ambiguity vector so obtained therefore read

$$
\begin{array}{ccc}
{\left[\hat{a}_{1}\right]} & = & {\left[\hat{a}_{1}\right]} \\
{\left[\hat{a}_{2 \mid 1}\right]} & = & {\left[\hat{a}_{2}-\sigma_{\hat{a}_{2} \hat{a}_{1}} \sigma_{\hat{a}_{1}}^{-2}\left(\hat{a}_{1}-\left[\hat{a}_{1}\right]\right)\right]} \\
\vdots & \vdots & \vdots \\
{\left[\hat{a}_{n \mid N}\right]=} & {\left[\hat{a}_{n}-\sum_{i=1}^{n-1} \sigma_{\hat{a}_{n} \hat{a}_{i \mid}} \sigma_{\hat{a}_{i \mid I}}^{-2}\left(\hat{a}_{i \mid I}-\left[\hat{a}_{i \mid I}\right]\right)\right]}
\end{array}
$$

where the shorthand notation $\hat{a}_{i \mid I}$ stands for the $i$ th leastsquares ambiguity obtained through a conditioning on the previous $I=\{1, \ldots,(i-1)\}$ sequentially rounded ambiguities. The integer bootstrapped solution therefore reads

$\breve{a}_{B}=\left(\left[\hat{a}_{1}\right], \ldots,\left[\hat{a}_{n \mid N}\right]\right)^{T}$

Note that this integer estimator is a generalization of the previous one [Eq. (12)]. The two integer estimators are identical in the case that the variance-covariance matrix is diagonal and they differ when this matrix is nondiagonal. In the non-diagonal case, however, the bootstrapped estimator still makes use of the simple 'integer rounding' operation, but now by taking the (sequential) correlations into account as well. The bootstrapped probability of correct integer estimation is given as

$P\left(\breve{a}_{B}=a\right)=P\left(\cap_{i=1}^{n}\left\{\left|\hat{a}_{i \mid I}-a_{i}\right| \leq \frac{1}{2}\right\}\right)$

In contrast with Eq. (13), this bootstrapped probability has a useful property in that it can be computed exactly in a rather straightforward manner. This follows from applying the chain rule of conditional probabilities

$$
\begin{gathered}
P\left(\breve{a}_{B}=a\right)=\prod_{i=1}^{n} P\left(\left[\hat{a}_{i \mid I}\right]=a_{i} \mid\left[\hat{a}_{1}\right]=a_{1},\right. \\
\left.\ldots,\left[\hat{a}_{(i-1) \mid(I-1)}\right]=a_{i-1}\right)
\end{gathered}
$$

Each of the probabilities in this chain is of the type of Eq. (11), but now with the ambiguity standard deviations replaced by the sequential conditional standard deviations. Hence, the bootstrapped probability of obtaining the correct integer ambiguity vector reads as

$P\left(\breve{a}_{B}=a\right)=\prod_{i=1}^{n}\left(2 \Phi\left(\frac{1}{2 \sigma_{\hat{a}_{i \mid}}}\right)-1\right)$

This probability can now be used to infer whether or not it makes sense to use the integer bootstrapped solution.
Note, that since each of the individual probabilities in the product of Eq. (19) is smaller than one, the overall probability has the tendency to get smaller as the dimension $n$ increases. Hence, for a particular application it could well be that the overall probability is too small, while the product of the first $j<n$ terms is still large enough to render successful fixing of the first $j$ ambiguities possible. In that case Eq. (19) can be used to study the success rate of a partial fixing of the ambiguities. Also note that the probability is not invariant against a reparametrization of the ambiguities. In fact, the bootstrapped solution and its corresponding probability are not even invariant against a reordering or permutation of the ambiguities. Hence, as was the case with the previous integer estimator, it also makes sense for the bootstrapped estimator to first transform the ambiguities to a new set of more precise ambiguities before commencing with the bootstrapping.

\subsection{The probability of bootstrapping and rounding}

The relative performance of the 'integer bootstrapping' estimator and the 'integer rounding' estimator can be evaluated if we compare their respective probabilities of correct integer estimation. Since the bootstrapped solution takes part of the correlation structure into account, whereas the solution obtained through rounding does not, one will be inclined to think that the bootstrapped probability of correct integer estimation is larger than or at least as large as the probability that corresponds with rounding. Hence, the conjecture is that the inequality

$P\left(\breve{a}_{R}=a\right) \leq P\left(\breve{a}_{B}=a\right)$

holds true. In order to prove this inequality we will start with the probability of correct rounding. It reads

$$
\begin{aligned}
P\left(\breve{a}_{R}=a\right)= & \int_{R} \frac{1}{(2 \pi)^{\frac{1}{2} n} \sqrt{\operatorname{det}\left(Q_{\hat{a}}\right)}} \\
& \times \exp \left\{-\frac{1}{2}(\hat{a}-a)^{\mathrm{T}} Q_{\hat{a}}^{-1}(\hat{a}-a)\right\} \mathrm{d} \hat{a}
\end{aligned}
$$

with the region of integration

$R=\left\{\left(\hat{a}_{1}, \ldots, \hat{a}_{n}\right)^{T} \in \mathbf{R}^{n}|| \hat{a}_{i}-a_{i} \mid \leq \frac{1}{2}, i=1, \ldots, n\right\}$

This region is a cube having all side lengths equal to 1 . The above integral is parametrized in terms of $\hat{a}_{i}$. In order to obtain a link with the bootstrapped probability of correct integer estimation, we will reparametrize the integral in terms of $\hat{a}_{i \mid I}$. The corresponding transformation reads

$\hat{a}_{i}=\hat{a}_{i \mid I}+\sum_{j=1}^{i-1} \sigma_{\hat{a}_{i} \hat{a}_{j \mid J}} \sigma_{\hat{a}_{j \mid J}}^{-2}\left(\hat{a}_{j \mid J}-a_{j}\right) \quad$ for $\quad i=1, \ldots, n$

In order to apply this transformation to the integral of Eq.(21), we need the general transformation formula for integrals. This reads (Fleming, 1977) 
$\int_{R} f(y) \mathrm{d} y=\int_{g^{-1}(R)} f(g(x))\left|\operatorname{det}\left(\partial_{x} g(x)\right)\right| \mathrm{d} x$

By letting Eq. (22) play the role of $y=g(x)$, we obtain, noting that the matrix of transformation (22) is unit triangular and therefore that the determinant of the Jacobian equals one

$$
\begin{aligned}
P\left(\breve{a}_{R}=a\right)= & \int_{g^{-1}(R)} \frac{1}{(2 \pi)^{\frac{1}{2} n} \prod_{i=1}^{n} \sigma_{\hat{a}_{i \mid I}}} \\
& \times \prod_{i=1}^{n} \exp \left\{-\frac{1}{2}\left(\frac{\hat{a}_{i \mid I}-a_{i}}{\sigma_{\hat{a}_{i \mid I}}}\right)^{2}\right\} \prod_{i=1}^{n} \mathrm{~d} \hat{a}_{i \mid I}
\end{aligned}
$$

with the region of integration

$g^{-1}(R)=\left\{\left(\hat{a}_{1}, \hat{a}_{2 \mid 1}, \ldots, \hat{a}_{n \mid N}\right)^{T} \in \mathbf{R}^{n} \mid S_{i}, i=1, \ldots, n\right\}$

where

$S_{i}=\left\{\left|\left(\hat{a}_{i \mid I}-a_{i}\right)+\sum_{j=1}^{i-1} \sigma_{\hat{a}_{i} \hat{a}_{j \mid J}} \sigma_{\hat{a}_{j \mid J}}^{-2}\left(\hat{a}_{j \mid J}-a_{j}\right)\right| \leq \frac{1}{2}\right\}$

This region of integration is now no longer a cube. However, due to the product form of the integral and the sequential form of the intervals in $g^{-1}(R)$, it follows that when we write the integral as

$$
\begin{aligned}
P\left(\breve{a}_{R}=a\right)= & \int_{g^{-1}(R) \mid S_{n}} F_{n} \prod_{i=1}^{n-1} \frac{1}{\sqrt{2 \pi} \sigma_{\hat{a}_{|| I}}} \\
& \times \exp \left\{-\frac{1}{2}\left(\frac{\hat{a}_{i \mid I}-a_{i}}{\sigma_{\hat{a}_{i \mid I}}}\right)^{2}\right\} \prod_{i=1}^{n-1} \mathrm{~d} \hat{a}_{i \mid I} \\
F_{n}= & \int_{S_{n}} \frac{1}{\sqrt{2 \pi} \sigma_{\hat{a}_{n \mid N}}} \exp \left\{-\frac{1}{2}\left(\frac{\hat{a}_{n \mid N}-a_{n}}{\sigma_{\hat{a}_{n \mid N}}}\right)^{2}\right\} \mathrm{d} \hat{a}_{n \mid N}
\end{aligned}
$$

the dependence on $\hat{a}_{n \mid N}$ is completely captured in $F_{n}$. We are now in a position to formulate an upper bound. Note that the interval $S_{n}$ is not centred at the mean $a_{n}$. Hence, this integral of the standard normal distribution will increase in value if the interval $S_{n}$ is replaced by the interval $B_{n}=\left\{\left|\hat{a}_{n \mid N}-a_{n}\right| \leq 1 / 2\right\}$. This shows that

$F_{n} \leq\left(2 \Phi\left(\frac{1}{2 \sigma_{\hat{a}_{n \mid N}}}\right)-1\right)$

By repeating these steps for the other variables also, we finally obtain the upper bound

$P\left(\breve{a}_{R}=a\right) \leq \prod_{i=1}^{n}\left(2 \Phi\left(\frac{1}{2 \sigma_{\hat{a}_{i \backslash I}}}\right)-1\right)$

which concludes the proof of Eq. (20). Note that when the upper bound Eq. (20), is combined with the lower bound, Eq. (14), we have an easy-to-compute interval that completely bounds the probability of correct integer rounding. To reduce the length of the interval, the bounds should be evaluated with the transformed and decorrelated ambiguities.

\subsection{An invariant upper bound}

We have already observed that the bootstrapped probability [Eq. (19)] depends on the chosen ambiguity parametrization. Hence, by using more precise transformed ambiguities instead of the DD ambiguities, one can increase the probability of correct integer estimation. Although this probability can be computed exactly for every chosen parametrization, it would still be helpful if we could come up with an upper bound which is easy to compute and at the same time invariant for the choice of parametrization. Such an upper bound can be found as follows. Note that Eq. (17) equals the integral of a normal distribution over an $n$-dimensional box. When the side lengths are taken equal to the reciprocal value of the sequential conditional standard deviations, the normal distribution takes its standard form of having zero mean and a unit variance-covariance matrix. This implies, if we vary the side lengths but constrain the volume of the box to be constant, that this probability reaches its maximum for a cube. Since this cube must have the same volume as the original box, its side lengths are all equal to the geometric average of the reciprocal sequential conditional standard deviations. Hence, we have

$$
\begin{aligned}
& P\left(\cap_{i=1}^{n}\left\{\frac{\left|\hat{a}_{i \mid I}-a_{i}\right|}{\sigma_{\hat{a}_{i \mid I}}} \leq \frac{1}{2 \sigma_{\hat{a}_{i \mid I}}}\right\}\right) \\
& \quad \leq P\left(\bigcap_{i=1}^{n}\left\{\frac{\left|\hat{a}_{i \mid I}-a_{i}\right|}{\sigma_{\hat{a}_{i \mid I}}} \leq \frac{1}{2} \sqrt[n]{\prod_{i=1}^{n} \frac{1}{\sigma_{\hat{a}_{i \mid}}}}\right\}\right)
\end{aligned}
$$

In this expression we recognize on the right-hand side the ADOP (Ambiguity Dilution of Precision) which was introduced by Teunissen (1997b) as

$$
\mathrm{ADOP}=\sqrt{\operatorname{det} Q_{a}}{ }^{\frac{1}{n}} \quad(\text { cycle })
$$

It measures the average precision of the ambiguities and it is invariant for the class of admissible ambiguity transformations (Teunissen and Odijk 1997). The above upper bound can thus be formulated in a compact way as

$P\left(\breve{a}_{B}=a\right) \leq\left(2 \Phi\left(\frac{1}{2 \mathrm{ADOP}}\right)-1\right)^{n}$

Since the ADOP is invariant for all admissible ambiguity transformations, this also holds true for the above upper bound. The upper bound can now be used, independently of which parametrization is used, to check whether it makes sense to consider the bootstrapping method as a viable option for integer estimation. The bootstrapping method should not be used when the upper bound is too small. Closed form expressions for the determinant of the ambiguity variance-covariance matrix are given by Teunissen (1997c). By means of 
these closed form expressions it becomes obvious in what way the various factors such as number of satellites tracked, number of observation epochs used, type of observables used and amount of change in relative receiver-satellite geometry, contribute to the above bound.

\section{Conclusions}

In this contribution we have studied two simple alternatives of the integer least-squares estimator. Although they are less optimal, they have the advantage that no search at all is needed for their actual computation. However, before the choice is made to opt for one of these two integer estimators, one should make sure that their probability of correct integer estimation is sufficiently close to one. This is especially of importance for GPS ambiguity resolution, where in all subsequent processing steps the integer ambiguity solution is treated as non-stochastic.

The first integer estimator considered was the one that corresponds to a componentwise rounding of the 'float' solution. Although it is difficult to compute its probability of correct integer estimation exactly, it was shown that this probability is bounded as

$$
\begin{gathered}
\prod_{i=1}^{n}\left(2 \Phi\left(\frac{1}{2 \sigma_{\hat{a}_{i}}}\right)-1\right) \leq P\left(\breve{a}_{R}=a\right) \\
\leq \prod_{i=1}^{n}\left(2 \Phi\left(\frac{1}{2 \sigma_{\hat{a}_{i \mid}}}\right)-1\right)
\end{gathered}
$$

Since the probability (as well as its two bounds) is dependent on the choice of ambiguity parametrization, one should apply this estimator only after the ambiguity decorrelation process has been applied.

The second integer estimator considered was the bootstrapped one. It follows from a sequential rounding of the entries of the 'float' solution. Since the variancecovariance matrix of the sequential conditional leastsquares solution is diagonal, the probability of correct integer estimation of the bootstrapped solution can be computed exactly. It was shown that

$$
\begin{aligned}
& \prod_{i=1}^{n}\left(2 \Phi\left(\frac{1}{2 \sigma_{\hat{a}_{i||}}}\right)-1\right)=P\left(\breve{a}_{B}=a\right) \\
& \leq\left(2 \Phi\left(\frac{1}{2 \mathrm{ADOP}}\right)-1\right)^{n}
\end{aligned}
$$

Hence, with the bootstrapped solution one has a better chance of obtaining the correct integer ambiguities. Here, the remark that the evaluation should be based on the reparametrized and decorrelated ambiguities again applies. Although the probability of correct integer estimation can be computed exactly for the bootstrapped solution, the above given upper bound is still very useful. First, it is invariant for the choice of ambiguity parametrization, and second, simple closedform expressions are available for evaluating the ADOP of the ambiguity variance-covariance matrix.

\section{References}

Blewitt G (1989) Carrier-phase ambiguity resolution for the Global Positioning System applied to geodetic baselines up to $2000 \mathrm{~km}$. J Geophys Res 94 (B8): 10.187-10.302

Boon F, Ambrosius B (1997) Results of real-time application of the LAMBDA method in GPS based aircraft landings. Proc. KIS97, pp 339-345

de Jonge PJ (1998) A processing strategy for the application of the GPS in networks. Netherlands Geodetic Commission, Publications on Geodesy, No. 46.

de Jonge PJ, Tiberius CCJM (1996) The LAMBDA method for integer ambiguity estimation: implementation aspects LGRSeries no. 12. Delft Geodetic Computing Centre, 49 pp

Dong D, Bock Y (1989) Global Positioning System network analysis with phase ambiguity resolution applied to crustal deformation studies in California. J Geophys Res 94: 3949-3966

Fleming W (1977) Functions of Several Variables. Springer, Berlin Heidelberg New York

Hofmann-Wellenhof B, Lichtenegger H, Collins J (1997) Global positioning system: theory and practice. 4th edn. Springer, Berlin Heidelberg New York

Johnson NL, Kotz S, Balakrishnan N (1994) Continuous univariate distributions, vol 1, 2nd edn. Wiley, New York

Jonkman NF (1998) Integer GPS ambiguity estimation without the receiver-satellite geometry. LGR-Series no. 18. Delft Geodetic Computing Centre, 95 pp

Kleusberg A, Teunissen PJG (eds) (1996) GPS for Geodesy. Lecture Notes in Earth Sciences, vol 60. Springer, Berlin Heidelberg New York

Strang G, Borre K (1997) Linear algebra, geodesy, and GPS. Wellesley-Cambridge Press, Cambridge, Massachusetts

Teunissen PJG (1993) Least-squares estimation of the integer GPS ambiguities. Invited Lecture, Sect IV theory and methodology, IAG general meeting, Beijing. Also in: LGR-Series no. 6. Delft Geodetic Computing Centre

Teunissen PJG (1995) The invertible GPS ambiguity transformations. Manusci Geod 20: 489-497

Teunissen PJG (1996) GPS carrier phase ambiguity fixing concepts. In: Kleusberg A, Teunissen PJG (eds) GPS for geodesy. Lecture Notes in Earth Sciences, vol 60. Springer, Berlin Heidelberg New York, pp 263-335

Teunissen PJG (1997a) Some remarks on GPS ambiguity resolution. Artificial Satellites 32(3), pp 119-130

Teunissen PJG (1997b) A canonical theory for short GPS baselines, Parts I-IV. J Geod 71: 320-336, 389-401, 486-501, 513-525

Teunissen PJG (1997c) Closed form expressions for the volume of the GPS ambiguity search spaces. Artif Satellites 32(1): 5-20

Teunissen PJG (1998) A class of unbiased integer GPS ambiguity estimators. Artif Satellites 33(1), pp 3-10

Teunissen PJG, Odijk D (1997) Ambiguity dilution of precision: definition, properties and application. Proc ION GPS-97, vol 1, pp 891-899

Teunissen PJG, de Jonge PJ, Tiberius CCJM (1996) The volume of the GPS ambiguity search space and its relevance for integer ambiguity resolution. Proc ION GPS-96, pp 889-898

Teunissen PJG, de Jonge PJ, Tiberius CCJM (1997) The performance of the LAMBDA method for fast GPS ambiguity resolution. Navigation 44(3): 373-383 